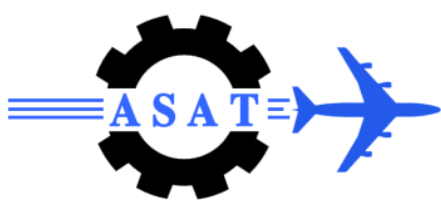

\title{
Parameter Identification of Finite Element Model Using a Genetic Algorithm
}

\begin{abstract}
S.Y. Mahfouz ${ }^{*}$
Abstract: The present paper discusses the ability of a developed optimization technique to obtain parameters that can be used in the simulation of a finite element model of 3D portal frame structure where a set of parameters that gives acceptable response of the structure is needed. In order to find the values of these parameters, an optimization technique based on a modified genetic algorithm (GA) was developed and linked to a finite element package. In the formulation of the optimization problem, the objective function is the differences between the computed structural response and that measured experimentally where the design variables describe the rotational stiffnesses of various connections of the bare frame, the steel sheet cladding thickness, the orthotropic properties of the cladding and the stiffness of the fasteners.
\end{abstract}

Keywords: Genetic Algorithm, Parameter Identification, Structural Optimization.

\section{Introduction}

Finite element analysis is an appropriate method for understanding the response of structures comprised of a large number of elements. Computer modelling of clad structures includes parameters representing the connections, cross sectional and material properties of the members forming the structure (i.e. bare frames, bracing system, purlins, girts, sheeting, cleats, fasteners). These parameters need to be identified. The method of identifying the model parameters is called model validation. A steel structure with profiled steel cladding was modelled using a full three-dimensional finite element analysis.

In order to deal with such case study, the features of structural problem are addressed: 1) some or all parameters can be defined as a set of discrete parameters, 2) the structure response (i.e. stresses, deformations) are implicit functions of those parameters, 3) these functions can only be estimated with a finite accuracy by means of finite element analysis. Therefore, various optimisation algorithms has been linked to the structural analysis software since early 1960s with varied degree of success. A study of this experience allows formulating the following requirements to a structural optimization technique. Firstly, the technique should be able to deal with realistic structural problem. Secondly, it has to require a minimum amount of auxiliary information to guide the search. Lastly, it shall attempt to reach the global optimum.

\footnotetext{
*: Egyptian Armed Forces, Egypt, E-mail: samehmahfouz@netscape.net 
In the present study, a genetic algorithm (GA) has been selected as a basis for the development of an efficient structural optimization algorithm because it satisfies the aforementioned requirements. Various aspects of genetic algorithms are discussed in details by many authors among them Goldberg (1989), Davis (1991), Xie et al. (1997) and Mitchell (1998).

A typical GA is a relatively slow technique as compared to the derivative-based ones. Therefore, modification to the basic procedure is needed in order to improve its rate of convergence

The modified genetic algorithm was utilized to obtain proper values for the unknown parameters to give good agreement between the displacements at different locations computed when using the finite element mathematical model and these measured during the structural tests.

\section{Genetic Algorithm}

A standard genetic algorithm (GA) was introduced by John Holland (1975) and is inspired by the observation of how adaptation takes place in evolving natural systems. Holland proposed searching a general space using reproduction plans which would selectively manipulate and reproduce a collection population of candidates solutions called individuals to generate new individuals on the most promising areas of the search space. In the simplest form of the GA, candidate solutions to some problem are encoded in binary strings which play the role of artificial chromosomes, while individual bits play the role of genes. Each individual therefore comprises a candidate solution to a specific problem and has a corresponding fitness.

A basic genetic algorithm consists of three main operators, namely, selection, crossover and mutation. In the selection operator, chromosomes in the population for reproduction are randomly selected. The fitter the chromosomes, the more times it is likely to be selected to reproduce. The reproduction operator may be implemented in a number of ways, the most popular being used is a biased roulette wheel with slots of different width representing the proportion of the fitness of an individual string or, in other words, quality of a solution. The crossover operator randomly chooses a locus and exchanges the subsequences before and after that locus between two chromosomes to create two offspring. The crossover operator can be applied using different techniques, the most common ones are single-point crossover, twopoint crossover and multi-point crossover. Lastly, in order to introduce new genetic patterns in the child strings, the mutation operator, which flips some of the bits in a randomly selected chromosomes takes place with a low probability thus preventing the search from premature convergence to a non-optimal solution and improving non-local properties of the search.

Generally, GA is judged to be successful if it evolves a population of highly fit individuals as a result of iterating this procedure through successive generations. More details regarding the various aspects of GA are discussed by many authors among them Goldberg (1989), Davis (1991), Holland (1992), Xie et al. (1997) and Singiresu (2009).

In the present paper, a strategy developed to transfere the best members of a current population to the next population. Then, a common pattern of the elite part has been established and imposed on all the remaining strings except for the elite. The remaining parts of strings in the rest of the population are filled by coping the rest of strings (not prescribed by the imposed pattern) from the strings in the previous population. After filling all the missing parts of the strings and fitness evaluation for newly obtained strings, the population is 
subjected to either uniform crossover or shuffle crossover presented by Eshelman et al. (1989), Syswerda (1989) and Mitchell (1998). The mutation stage is implemented using the following technique.

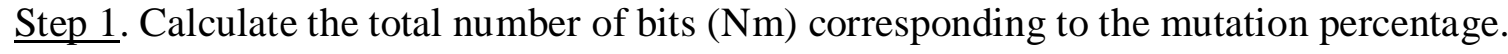

Step 2. Select randomly a bit in a chromosome.

Step 3. Change the bit to 0 , if it is 1 . Otherwise, keep the bit as it is.

$\underline{\text { Step } 4}$. Repeat steps 2 and 3 till the total number of bits $\left(N_{m}\right)$ is reached

The question of how to get an equal probability of selection for all discrete values existing in each design space of each design variable is answered when using the following technique.

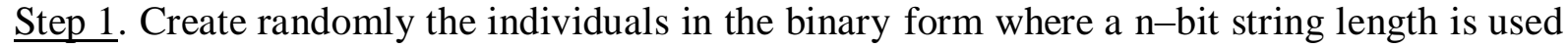
for each design variable.

Step 2. Calculate the integer number corresponds to the binary form for each design variable separately. This number should be less than or equal to $2^{n}$.

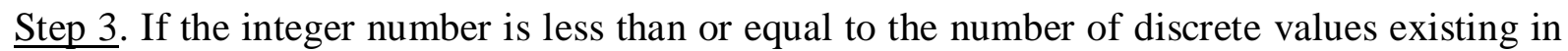
the design space $\left(x^{\mathrm{d}}\right)$, then select the value of the design variable corresponding to that number.

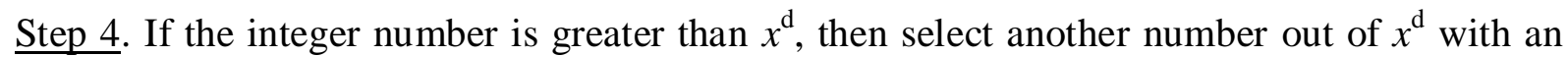
equal probability of selection of all numbers (from $1-x^{\mathrm{d}}$ ). This results in changing the chromosomes of the corresponding design variable,

$\underline{\text { Step } 5}$. Convert the selected integer number in step 4 to the binary form using the $2^{n}$-bit string length.

$\underline{\text { Step } 6}$. Replace the binary strings selected in step 1 by those obtained in step 5 .

\section{Test Problem: Ten-Bar Truss}

The testing of the developed technique has been carried out on a standard problem, see Rajeev and Krishnamoorthy (1992), of a ten-bar truss shown in Fig. 1. Stresses have been limited by $\pm 25 k s i$ in all members. The vertical displacements at nodes $\mathrm{D}$ and $\mathrm{E}$ have been limited to 2 $i n$. The modulus of elasticity $E$ for all truss members is $10^{7} \mathrm{psi}$. The density of the truss material $\rho_{\text {den }}$ equals $0.1 \mathrm{lb} / \mathrm{in}^{3}, L_{n}$ mem and $x_{n \text { mem }}$ are the length and cross sectional area of the element numbered $n^{\text {mem }}$ respectively. Design variables are allowed to take discrete values in the design space $D$ from the AISC manual listed in. $D$ contains discrete values $(1.62,1.8$, $1.99,2.13,2.38,2.62,2.63,2.88,2.93,3.09,3.13,3.38,3.47,3.55,3.63,3.84,3.87,3.88$, $4.18,4.22,4.49,4.59,4.80,4.97,5.12,5.74,7.22,7.97,11.5,13.5,13.9,14.2,15.5,16.0$, $16.9,18.8,19.9,22.0,22.9,26.5,30.0,33.5 \mathrm{in}^{2}$ ). Here the number of available values for each design variable is 42 . However, for optimization process to take place via the GA, this number has to be accommodated in a 6-bit string length, which results in 22 vacant positions. This results in overall string length of 60 .The optimization problem can therefore be formulated as:

$$
\begin{gathered}
\text { Minimize } F(\boldsymbol{x})=\rho_{\mathrm{den}} \sum_{n^{\mathrm{mem}}=1}^{10} x_{n^{\mathrm{mem}}} L_{n^{\mathrm{mem}}}, \\
\text { subject to: } \frac{G_{s}(\boldsymbol{x})}{\widetilde{G}_{s}} \leq 1, s=1,2, \cdots 12 \\
x_{n^{\mathrm{mem}}} \in D \text { and } n^{\mathrm{mem}}=1,2, \cdots 10
\end{gathered}
$$


where $G_{s}(\boldsymbol{x})$ describes the computed stresses and nodal displacements. $\widetilde{G}_{s}(\boldsymbol{x})$ indicates the allowable stresses and nodal displacements.

The results obtained are compared with the solutions given by Rajeev and Krishnamorthy (1992). This comparison is introduced in Table 1a. Table 1b represents the values of the nodal displacement at the best solution. The convergence history is graphically depicted in Fig.2.

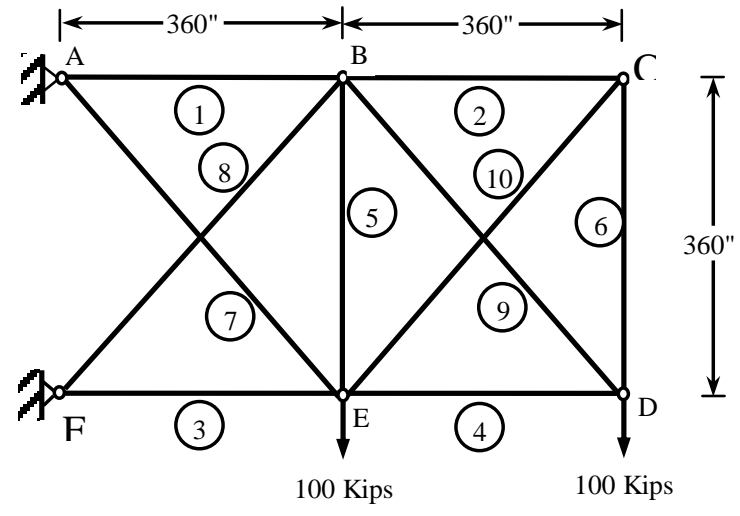

Fig. 1. Ten-bar truss

Table 1a. Comparison of the best solutions

\begin{tabular}{|c|c|c|c|c|c|}
\hline \multirow{3}{*}{$\begin{array}{c}\text { Truss } \\
\text { member }\end{array}$} & \multirow{3}{*}{$\begin{array}{c}\text { Rajeev and } \\
\text { Krishn-amorthy } \\
(1992) \\
\text { Area }\left(i n^{2}\right)\end{array}$} & \multicolumn{4}{|c|}{ Present study } \\
\hline & & \multicolumn{2}{|c|}{ uniform crossover } & \multicolumn{2}{|c|}{ shuffle crossover } \\
\hline & & Area $\left(i n^{2}\right)$ & Stress $(k s i)$ & Area $\left(i n^{2}\right)$ & Stress $(k s i)$ \\
\hline 1 & 33.50 & 33.5 & 6.58 & 33.5 & 6.603 \\
\hline 2 & 1.62 & 1.62 & 1.06 & 1.62 & 1.107 \\
\hline 3 & 22.00 & 22.9 & -7.83 & 22.9 & -7.807 \\
\hline 4 & 15.50 & 15.5 & -6.34 & 14.2 & -6.916 \\
\hline 5 & 1.62 & 1.62 & 13.85 & 1.62 & 14.197 \\
\hline 6 & 1.62 & 1.62 & 1.06 & 1.62 & 1.107 \\
\hline 7 & 14.2 & 7.97 & 14.07 & 7.97 & 13.981 \\
\hline 8 & 19.9 & 22.0 & -7.76 & 22.9 & -7.485 \\
\hline 9 & 19.9 & 22.0 & 6.318 & 22.0 & 6.313 \\
\hline 10 & 2.62 & 1.62 & -1.50 & 1.62 & -1.565 \\
\hline Weight $(l b)$ & 5613.8 & \multicolumn{2}{|c|}{5491.717} & \multicolumn{2}{|c|}{5490.737} \\
\hline
\end{tabular}

Table 1b. The displacement values of nodes at the best solution

\begin{tabular}{c|c|c}
\hline \hline \multirow{2}{*}{ Truss nodes } & \multicolumn{2}{|c}{ Constraint value (displacement in) } \\
\cline { 2 - 3 } & Using uniform crossover & Using shuffle crossover \\
\hline D & 1.998115 & 1.99894 \\
\hline E & 1.294658 & 1.28774 \\
\hline
\end{tabular}




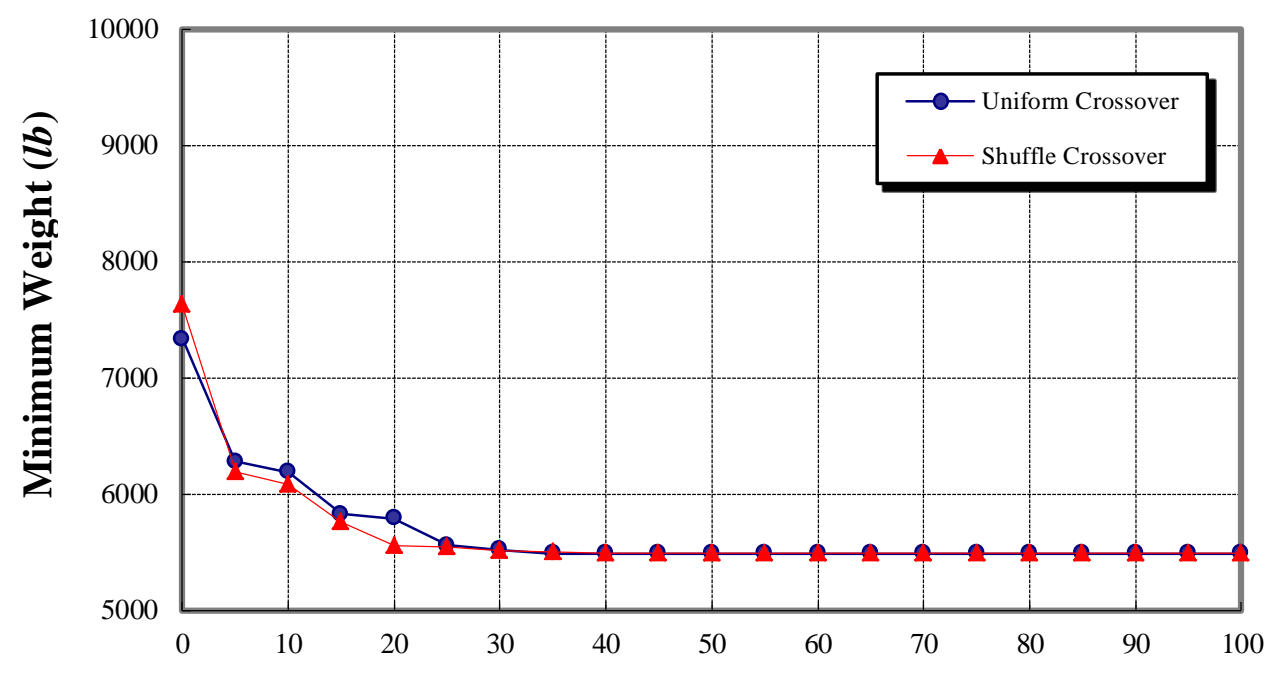

Generation Number

Fig. 2. Ten-bar truss: generation number versus minimum weight

\section{The Steel Clad Structure}

The steel portal framed clad structure (see, Warrior (1990) shown in Fig. 3 is investigated. The structure consists of two portal frames having 5.334 meters apart. The portal frame has a span of 13.716 meters. The sectional properties of the sheeting rail, eaves beam, purlin and corrugated sheets are also shown in Fig.3. The cladding sheets are $1 \mathrm{~m}$ wide, with ridges at $250 \mathrm{~mm}$ centres and $0.6 \mathrm{~mm}$ thick. The drilling screws of $4.2 \mathrm{~mm}$ diameter are used to secure cladding panels to purlins and sheeting rails. The structure was investigated for five cases of base movements. Case 1, 2 and 3 are a $50 \mathrm{~mm}$ vertical movement, a $100 \mathrm{~mm}$ non-symmetrical compressive movement and a $100 \mathrm{~mm}$ non-symmetrical tensile movement respectively, all applied to the front left-hand base. Case 4 and 5 are the $100 \mathrm{~mm}$ symmetrical compressive and tensile movements respectively and these are imposed on both front bases of the structure.

The finite element modelling, using ANSYS 5.4, of the steel clad structure is performed as follows: 1) The bases are defined as pinned with different linear rotational stiffness in three dimensions. 2) The relative horizontal displacement between the end plates of the apex connection are modelled by providing a horizontal longitudinal spring between the end plates of the apex connection. 3) The stanchion and rafter were modelled using a symmetrical crosssection beam element, BEAM4 element type, 4) The BEAM44 element was used to model sheeting rail, purlin, eaves beam and haunch section. 5) The uniaxial tension-compression element type, LINK8, was defined for the cross bracing. 6) The base, sheeting rail, eaves beam, purlin and apex connections were simulated as pins with linear rotational stiffness using the COMBIN14 element. 7) The cladding panel sheets were modelled using the available rectangular shell element named SHELL63. 8) The fasteners and the rivets are included in the mathematical clad model using COMBIN14 element in order to simulate fasteners and rivets as springs having longitudinal stiffness in three directions. 


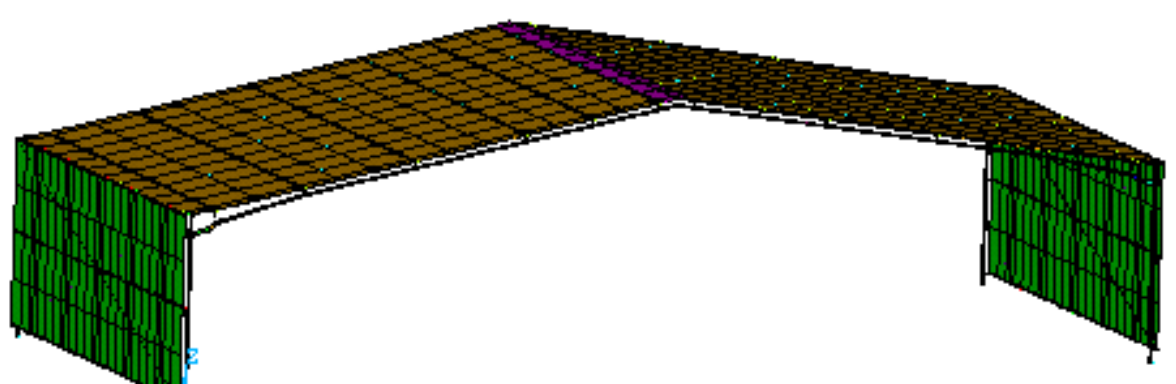

a) Isometric view
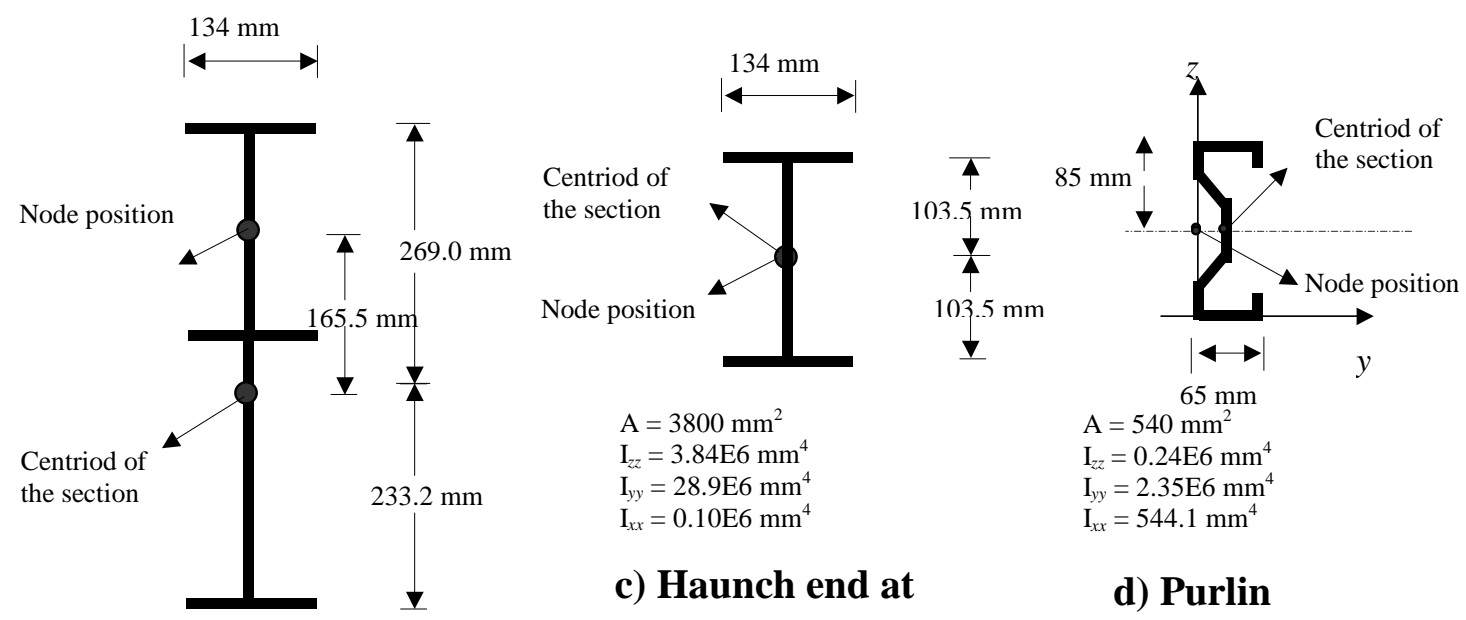

c) Haunch end at apex

$$
\begin{aligned}
\mathrm{A} & =7722 \mathrm{~mm}^{2} \\
\mathrm{I}_{z z} & =8.35 \mathrm{E} 6 \mathrm{~mm}^{4} \\
\mathrm{I}_{y y} & =259 \mathrm{E} 6 \mathrm{~mm}^{4} \\
\mathrm{I}_{x x} & =0.22 \mathrm{E} 6 \mathrm{~mm}^{4}
\end{aligned}
$$

b) Haunch end at stanchion

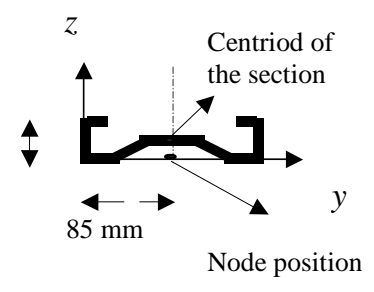

$\mathrm{A}=506 \mathrm{~mm}^{2}$

$\mathrm{I}_{z z}=2.07 \mathrm{E} 6 \mathrm{~mm}^{4}$

$\mathrm{I}_{y y}=0.14 \mathrm{E} 6 \mathrm{~mm}^{4}$

$\mathrm{I}_{x x}=506.2 \mathrm{~mm}^{4}$

\section{e) Sheeting Rail}

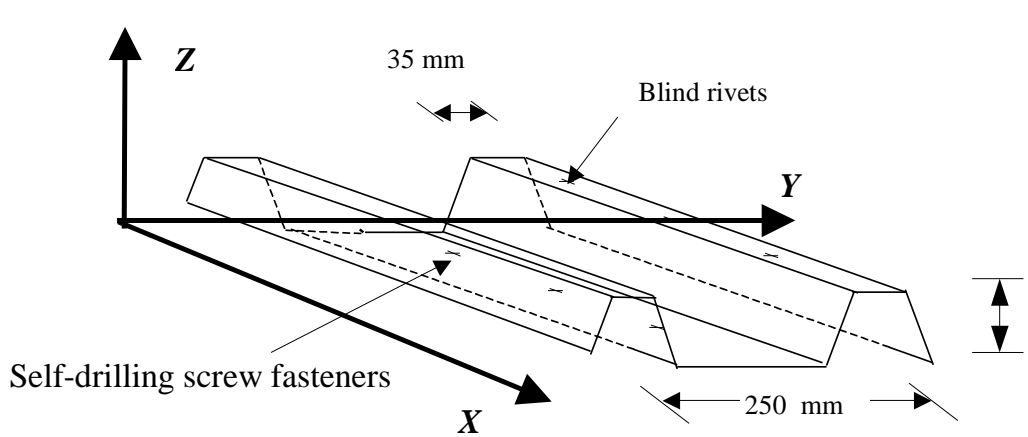

g) Corrugated sheets d) Purlin

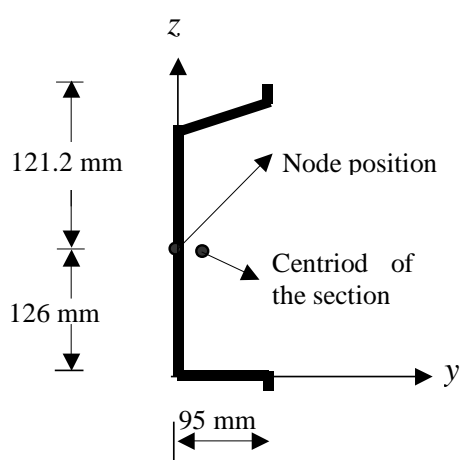

$\mathrm{A}=780 \mathrm{~mm}^{2}$

$\mathrm{I}_{z z}=0.75 \mathrm{E} 6 \mathrm{~mm}^{4}$

$\mathrm{I}_{\mathrm{yy}}=5.54 \mathrm{E} 6 \mathrm{~mm}^{4}$

$\mathrm{I}_{x x}=1120 \mathrm{~mm}^{4}$

f) Eaves beam

Fig. 3. The geometry of the steel portal framed clad structure 
In the Definition of the Optimization Problem, five objective functions are considered individually and collectively. The general form of the optimization problems can be formulated as follows:

$$
\begin{gathered}
\text { Minimize: } F(\boldsymbol{x})=\sum_{i=1}^{5} \boldsymbol{W}_{X} F_{X}^{i}(\boldsymbol{x})+W_{Y} F_{Y}^{i}(\boldsymbol{x})+W_{Z} F_{Z}^{i}(\boldsymbol{x}) \\
\text { Subject to: } \boldsymbol{x}=\left(\boldsymbol{x}_{1}^{T}, \boldsymbol{x}_{2}^{T}, \boldsymbol{x}_{j}^{T}, \cdots, \boldsymbol{x}_{J}^{T}\right), j=1,2,, \cdots, J \\
x_{j, k} \in D_{j}, j=1,2,3, \cdots, 20 \\
\text { and } D_{j}=\left(d_{j, 1}, d_{j, 2}, \cdots, d_{j, \ell}\right)
\end{gathered}
$$

where index $i$ define the vertical, left-hand compressive, left-hand tensile, symmetrical compressive and symmetrical tensile base movements respectively. The vector of design variables $\boldsymbol{x}$ is divided into $J$ sub-vectors $\boldsymbol{x}_{j}$. The components $x_{j, k}$ takes values from the corresponding domain $D_{j}$ where the number of values in each domain is $\ell$. The weighting coefficients $W_{X}, W_{Y}$ and $W_{Z}$ are taken; $W_{X}=1, W_{Y}=0.01$ and $W_{Z}=100$ (see, Chapman $e t$ al., 1999). The components of the objective function $\left(F_{X}^{i}(x), F_{Y}^{i}(x)\right.$ and $\left.F_{Z}^{i}(x)\right)$ describe the total difference of displacement between the measured and the computed values in $X, Y$ and $Z$ directions at all $n$ points along the front frame $(n=13)$. Each of the individual terms, ( $F_{X}^{i}(x), F_{Y}^{i}(x)$ and $\left.F_{Z}^{i}(x)\right)$ of the objective function, in the equation above, is calculated as shown in the following example:

$$
F_{X}^{i}(\boldsymbol{x})=\sum_{m=1}^{n}\left[\left(\hat{u}_{m}^{i}-u_{m}^{i}(\boldsymbol{x})\right) / \hat{u}_{m}^{i}\right]^{2},
$$

where the symbol ${ }^{\wedge}$ indicates the measured displacement components. The other components and their terms were defined in a similar manner.

The bare frame model contains 16 known parameters describing the various structural joints as springs. The values of these parameters are taken from Chapman et al. (1999). The cladding panels are modeled using 28 parameters representing the equivalent panel thicknesses, the orthotropic properties of the cladding and the stiffness of the screw fasteners and blind rivets. The parameters of the longitudinal stiffnesses are $\phi_{X}^{f, w}, \phi_{Y}^{f, w}$ and $\phi_{Z}^{f, w}$ for the wall fasteners connecting the cladding to the sheeting rails in the $\mathrm{X}, \mathrm{Y}$ and $\mathrm{Z}$ direction respectively; $\phi_{X}^{f, r}, \phi_{Y}^{f, r}$ and $\phi_{Z}^{f, r}$ represent longitudinal stiffnesses for the roof fasteners connecting the cladding to the purlins; $\phi_{X}^{r i, w}, \phi_{Y}^{r i, w}$ and $\phi_{Z}^{r i, w}$ describe longitudinal stiffnesses for the wall blind rivets connecting the cladding sheets together; $\phi_{X}^{r i, r}, \phi_{Y}^{r i, r}$ and $\phi_{Z}^{r i, r}$ are longitudinal stiffnesses for the roof blind rivets connecting the cladding sheets together; $E_{X}^{e q u i, w}, E_{Y}^{e q u i, w}$ and $E_{Z}^{e q u i, w}$ symbolize the Young's Modulii for the equivalent wall cladding panel; $E_{X}^{\text {equi,r }}, E_{Y}^{\text {equi,r }}$ and $E_{Z}^{\text {equi,r }}$ express the Young's Modulii for the equivalent roof cladding panel; $t^{e q u i, w}$ and $t^{e q u i, r}$ are the thicknesses of the equivalent wall and roof sheets respectively; $v_{X}^{\text {equi,w }}, v_{Y}^{\text {equi,w }}$ and $v_{Z}^{\text {equi,w }}$ are Poisson's ratios for the equivalent wall cladding panel; $v_{X}^{\text {equi,r }}, v_{Y}^{\text {equi, } r}$ and $v_{Z}^{\text {equi, } r}$ are Poisson's ratios for the 
equivalent roof cladding panel; $G^{e q u i, w}$ and $G^{\text {equi, } r}$ are the shear Modulii of the equivalent wall and roof cladding panels respectively. As a result of the parametric study presented by Chapman and Alqedra (1999) who studied the influence of these parameters on the structure response, these parameters are divided into two groups. The first, which have an influence more than $10 \%$ are considered unknown parameters. These are 15 unknown parameters; $\left(t^{e q u i, w}, t^{e q u i, r}, E_{X}^{e q u i, r}, \phi_{X}^{f, w}, \phi_{Y}^{f, w}, \phi_{Z}^{f, w}, \phi_{X}^{f, r}, \phi_{Y}^{f, r}, \phi_{Z}^{f, r}, \phi_{X}^{r i, w}, \phi_{Y}^{r i, w}, \phi_{Z}^{r i, w}\right.$,

$\phi_{X}^{r i, r}, \phi_{Y}^{r i, r}$ and $\left.\phi_{Z}^{r i, r}\right)$. The rest of the parameters take values presented by Chapman and Alqedra (1999). Upper and lower limits are specified to define the range of variation for the design variables. These limits are given in Table 2 . The string length corresponding to each design variable is given in Table 2. The overall string length is 202 .

Here, the optimization technique is linked to the finite element package ANSYS in order to compute the displacements at different nodes. The optimization search was carried out for each of the objective function component individually and the results obtained for the optimization variables for each base movement are presented in Table 3. The optimization process was also carried out including all five imposed base movements together (i.e. the objective function as defined by equation (2)) to determine one general set of values to be used in the model and gave reasonable agreement for all base movements. The results of what was named to be the overall model are also given in Table 3.

A comparison between the actual displacements and the displacements, computed from the case at which all the base movements acting together, is illustrated Fig. 4 indicating a very good agreement with those published Warrior (1990). It can be observed that the difference between the obtained values of the two longitudinal springs representing the in-plane stiffness in $x$ and $y$ directions of a fastener is very small. This agrees with Davies and Bryan (1982) who suggested modeling a fastener as a pair of springs having equal stiffness in two orthogonal directions but with no rotational stiffness. The differences between the values of the longitudinal stiffnesses for the roof and wall blind rivets are very small. This agrees with the parametric study presented by Chapman and Alqedra (1999).

\section{Conclusions}

Based on the present study, it can be concluded that:

1- The suggested modification to genetic algorithm to helps to find better solution for the tested problem.

2- The proposed optimization technique can be used to deal with a case study where the number of values in a domain of any design variable does not fit into a string.

3- Application to model validation of comprehensive model of a profiled clad portal frame structure is presented to demonstrate the potential application of the modified GA approach to complicated optimization problems in the field where there is no requirement not only for feasible initial solution but also for sensitivity analysis. 
Table 2. Upper and lower values of the variables of cladding panel

\begin{tabular}{c|c|c|c}
\hline Parameter symbol & String length & Lower limit $x_{i}^{\mathrm{L}}$ & Upper limit $x_{i}^{\mathrm{U}}$ \\
\hline $\begin{array}{c}\phi_{X}^{f, w}, \phi_{Y}^{f, w}, \phi_{Z}^{f, w}, \phi_{X}^{f, r}, \phi_{Y}^{f, r}, \\
\phi_{Z}^{f, r}, \phi_{X}^{r i, w}, \phi_{Y}^{r i, w}, \phi_{Z}^{r i, w}, \phi_{X}^{r i, r}, \\
\phi_{Y}^{r i, r} \text { and } \phi_{Z}^{r i, r}(\mathrm{kN} / \mathrm{m})\end{array}$ & 14 & & \\
\hline$E_{X}^{\text {equi,r }}\left(\mathrm{kN} / \mathrm{m}^{2}\right)$ & 22 & $20 \times 10^{7}$ & $10 \times 10^{4}$ \\
\hline$t^{\text {equi,w }}(\mathrm{mm})$ & 6 & 1 & $50 \times 10^{7}$ \\
\hline$t^{\text {equi,r }}(\mathrm{mm})$ & 6 & 1 & 50 \\
\hline \hline
\end{tabular}

Table 3. Values of the parameters of the comprehensive clad model corresponding to the individual base movements and to the overall comprehensive clad model.

\begin{tabular}{c|c|c|c|c|c|c}
\hline \multirow{2}{*}{$\begin{array}{c}\text { Parameter } \\
\text { symbol }\end{array}$} & \multicolumn{5}{|c|}{ Individual base movement modes } & \multirow{2}{*}{$\begin{array}{c}\text { All cases } \\
\text { acting } \\
\text { together }\end{array}$} \\
\cline { 2 - 6 } & Case 1 & Case 2 & Case 3 & Case 4 & Case 5 & 47 \\
\hline$t^{e q u i, w}$ & 2 & 34 & 25 & 38 & 39 & 2 \\
\hline$t^{e q u i, r}$ & 29 & 4 & 2 & 3 & 3 & $490 \times 10^{6}$ \\
\hline$E_{X}^{e q u i, r}$ & $285 \times 10^{6}$ & $427 \times 10^{6}$ & $476 \times 10^{6}$ & $135 \times 10^{6}$ & $142 \times 10^{6}$ & 452 \\
\hline$\phi_{X}^{f, r}$ & 176 & 333 & 486 & 25455 & 25452 & 352 \\
\hline$\phi_{Y}^{f, r}$ & 184 & 320 & 497 & 25429 & 25442 & 351 \\
\hline$\phi_{Z}^{f, r}$ & 302 & 110 & 568 & 5563 & 5563 & 503 \\
\hline$\phi_{X}^{f, w}$ & 26652 & 31861 & 79263 & 4285 & 4283 & 414 \\
\hline$\phi_{Y}^{f, w}$ & 26440 & 31774 & 79351 & 4290 & 4264 & 412 \\
\hline$\phi_{Z}^{f, w}$ & 74366 & 95501 & 93204 & 80184 & 80176 & 522 \\
\hline$\phi_{X}^{r i, r}$ & 3641 & 97241 & 1823 & 80235 & 80211 & 34061 \\
\hline$\phi_{Y}^{r i, r}$ & 3646 & 97250 & 1828 & 80246 & 80152 & 34123 \\
\hline$\phi_{Z}^{r i, r}$ & 3633 & 97230 & 1830 & 80239 & 80086 & 34199 \\
\hline$\phi_{X}^{r i, w}$ & 37354 & 77866 & 38322 & 88002 & 87903 & 19693 \\
\hline$\phi_{Y}^{r i, w}$ & 37242 & 78002 & 38401 & 87963 & 87908 & 19681 \\
\hline$\phi_{Z}^{r i, w}$ & 37189 & 77907 & 38299 & 87751 & 87799 & 19785 \\
\hline
\end{tabular}




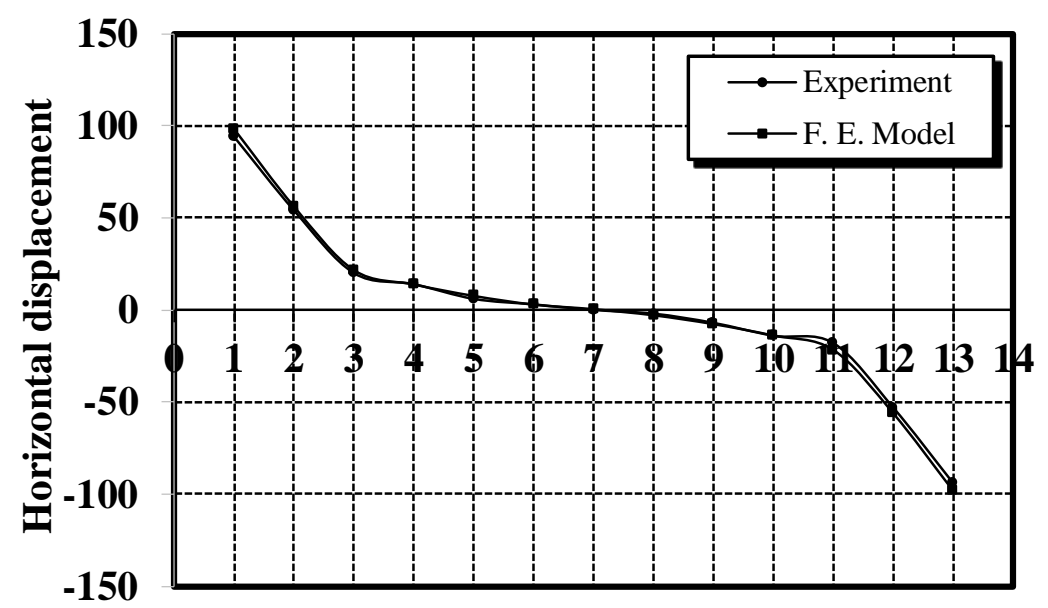

The point number along the frame

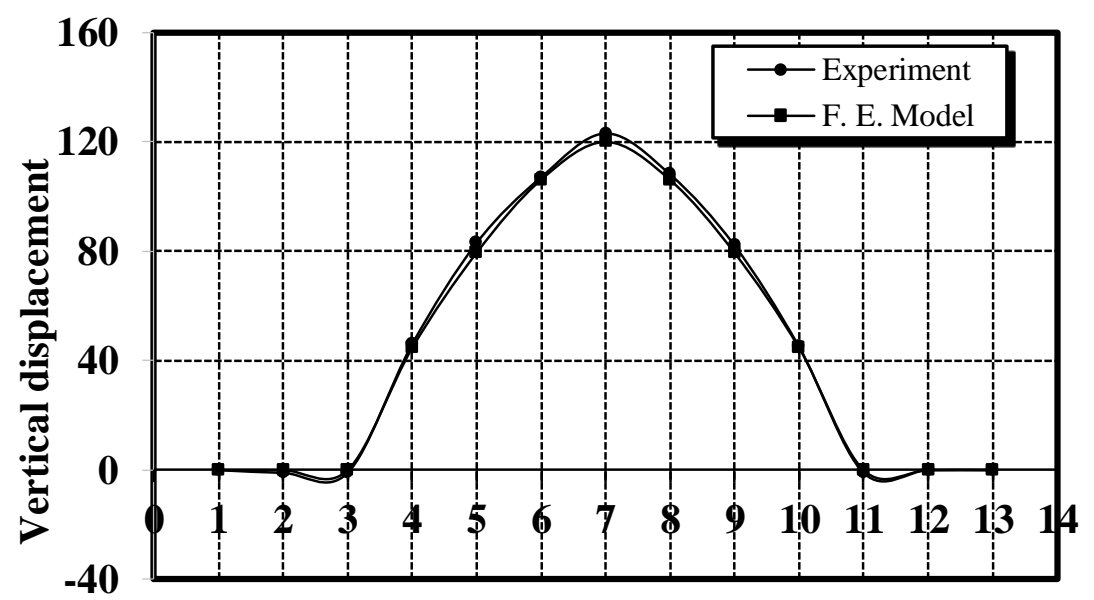

The point number along the frame



The point number along the frame

Fig. 4. A comparison between the actual and computed displacements for the case of the five base movements collectively 


\section{References}

[1] Ansys User's Manual (1996), Version 5.4, Swanson Systems Co.

[2] Chapman, B. C and Alqedra, M. A. (1999), "Use of optimization techniques for validation of finite element model of steel structure with profiled steel cladding", in: Friswell, M.I.; Mottershead, J.E. (Eds), Proceedings of International Conference on design optimisation $1^{\text {st }}$ ASMOUK, Ilkly, July 1999.

[3] Chapman, B. C, Alqedra, M. A. and Toropov, V. V. (1999), "Validation of threedimensional model of steel portal framed structure", in: Friswell, M.I.; Mottershead, J.E. (Eds), Proceedings of International Conference on Identification in Engineering Systems, Swansea, March 1999, pp261-270, The Cromwell Press Ltd, Broughton Gifford, Wiltshire.

[4] Davies, J. M. and Bryan, E. R., (1982), “Manual of Stressed Skin Diaphragm Design”, Granada Publishing Limited, Great Britain.

[5] Davis, L. (1991), "Handbook of Genetic Algorithms", Van Nostrand Reinhold, New York.

[6] Eshelman, L.J., Caruana, R. A. and Schaffer, J. D. (1989), "Biases in the crossover landscape", Proceeding of the $3^{\text {rd }}$ Genetic Algorithms Conference, pp. 11-19, San Mateo, California: Morgan Kaufman Publishers, Inc.

[7] Goldberg, D.E. (1989), "Genetic Algorithms in Search, Optimization and Machine Learning", Addison-Wesley, Reading, MA.

[8] Holland, J.H. (1975), "Adaptation in Natural and Artificial Systems", University of Michigan, Ann Arbor.

[9] Holland, J.H. (1992), "Adaptation in Natural and Artificial Systems. An Introductory Analysis with Applications to Biology, Control and Artificial Intelligence", MIT press.

[10] Mitchell, M (1998), "An Introduction to Genetic Algorithms", MIT Press, Cambridge, England.

[11] Moor, C., Mahendran, M., (1995), "Three-dimensional Analysis of Steel Portal Frame Buildings", in: Beasley, A. J., Foster, C. G. and Melerski, E. S. (Eds), Proceeding of the $14^{\text {th }}$ Australasian Conference on the Mechanics of Structures and Materials, Hobart, Australia, December 1995, pp.176-181.

[12] Rajeev, S. and Krishnamoorthy, C. S. (1992), "Discrete optimization of structures using genetic algorithms", Journal of Structural Engineering, ASCE, Vol 118, No. 5, pp. 1233-1250.

[13] Segerlind, L.J. (1984), "Applied Finite Element Analysis", John Wiley \& sons, New York.

[14] Singiresu, S. R., (2009), "Engineering Optimization: Theory and Practice ", John Wiley \& sons, New Jersy.

[15] Syswerda, G. (1989), "Uniform crossover in genetic algorithms", Proceeding of the $3^{\text {rd }}$ International Conference on Genetic Algorithms, pp. 2-9, Morgan Kaufman Publisher, Inc., CA.

[16] Warrior, D.A, (1990), "The Effects of Longwall Mining Subsidence on Steel Framed Buildings", Ph.D. Thesis, University of Bradford.

[17] Xie, Y.M., and Steven, G.P. (1997), "Evolutionary Structural Optimization", Springer, London. 